\title{
Parallel study on removal efficiency of pharmaceuticals and PFASs in advanced water treatment processes: Ozonation, GAC adsorption, and RO processes
}

\author{
Hyun-Seok Choe ${ }^{1}$, Ki Yong Kim ${ }^{2}$, Jeong-Eun $\mathrm{Oh}^{1}$, Jae-Hyuk Kim ${ }^{1^{\dagger}}$ \\ ${ }^{1}$ Department of Civil and Environmental Engineering, Pusan National University, Busan 46241, Republic of Korea \\ ${ }^{2}$ Food Safety and Processing Research Division, National Institute of Fisheries Science (NIFS), Busan 46083, Republic of Korea
}

\begin{abstract}
We aimed to assess the removal efficiencies of four pharmaceuticals (carbamazepine, crotamiton, metformin, and sulfamethoxazole) and four poly- and perfluoroalkyl substances (PFASs) (PFHxA, PFHxS, PFOA, and PFOS) by lab-scale ozonation, granular activated carbon (GAC) adsorption, and reverse osmosis (RO) membrane processes under varying operating conditions. Ozonation and GAC adsorption processes were conducted at two temperatures $\left(5\right.$ and $\left.25^{\circ} \mathrm{C}\right)$ and three $\mathrm{pH}$ conditions $(3,7$, and 11$)$. The membrane process was performed using an unstirred cell with two different RO membranes. The most pharmaceuticals were effectively removed by ozonation, whereas metformin and PFASs were unaffected due to their stable chemical structures. In the GAC process, metformin was hardly removed under acidic conditions but it was enhanced by over $90 \%$. PFASs were effectively removed by GAC adsorption and RO membrane processes. The RO membrane for brackish water treatment showed higher rejection than that for residential water treatment. Moreover, the rejection of PFAS increased as the molecular weight increased. A strategy was found to effectively remove the remaining metformin in most advanced water treatment processes. Chemically persistent PFASs were hardly removed by the ozonation process but were effectively removed by physical treatments such as GAC adsorption and RO membrane processes.
\end{abstract}

Keywords: GAC adsorption process, Membrane process, Ozonation process, Perfluoroalkyl substances, Pharmaceuticals

\section{Introduction}

The increasing production, consumption, and disposal of chemical products have raised public concern due to the hazard they pose to human health and the ecosystem [1-3]. The latest analytical technology has revealed that traces of contaminants are present even in purified tap water, which amplifies people's concerns [4-7]. Micropollutants (MPs) are anthropogenic pollutants of aquatic systems at low concentrations and represent a class of fastest emerging contaminants. They have been frequently detected in purified water because conventional drinking water treatment plants are not designed to treat MPs. MPs include pharmaceuticals, personal care products, poly- and perfluoroalkyl substances (PFASs), steroids, surfactants, industrial chemicals, pesticides, and heavy metals [8]. Among them, pharmaceuticals and PFASs have drawn enormous attention due to their bioactive properties and persistence, posing

This is an Open Access article distributed under the terms of the Creative Commons Attribution Non-Commercial License (http://creativecommons.org/licenses/by-nc/3.0/) which permits unrestricted non-commercial use, distribution, and reproduction in any medium, provided the original work is properly cited.

Copyright (C) 2022 Korean Society of Environmental Engineers potential risks to the environment and human health [9-13].

Pharmaceuticals are the substances used to prevent, diagnose, and treat diseases, and their types and forms can be as diverse as their purpose. Even though pharmaceuticals address health problems and as a result have improved longevity, indiscriminate exposure to pharmaceuticals can have unintended side-effects in the short and long term. Most of them are toxic to aquatic systems and can cause deformity $[10,14]$. In particular, stimulants (caffeine), antibiotics (sulfamethoxazole, clarithromycin), anticonvulsants (carbamazepine), skin itch treatments (crotamiton), diabetes management drugs (metformin), and anti-inflammatory drugs (naproxen) were frequently detected at high concentrations [15, 16].

PFASs refer to substances in which a part or all of the hydrogen $(\mathrm{H})$ in the hydrocarbon is substituted with fluorine $(\mathrm{F})$. The use of PFASs is widespread - from household products to industrial proposes - owing to their high durability, chemical resistance, oil
Received September 15, 2020 Accepted December 15, 2020

${ }^{\dagger}$ Corresponding author

E-mail: jaehyuk.kim@pusan.ac.kr

Tel: +82515102312

ORCID: 0000-0002-3215-2178 
resistance, and lubricity induced by strong C-F bonds [17]. However, these properties result in severe persistence, and when they leak into the ecosystem and body, they rarely dissolve [11, 13]. Accordingly, many countries have restricted the use of PFASs such as perfluorooctanoic acid (PFOA) and perfluorooctanesulfonic acid (PFOS) but other PFAS derivatives such as perfluorohexanoic acid (PFHxA) and perfluorohexanesulfonic acid (PFHxS) are still widely used and detected in aquatic systems [4, 16, 18]. In particular, in 2018, there were cases in which PFASs were detected in wastewater, treated water, water treatment plants, and tap water; this has, thus, become a social issue [5].

Thus, improved technologies are required to effectively treat such MPs, particularly for pharmaceuticals and PFASs. Advanced water treatment processes are physical/chemical treatment processes that can effectively remove these chemicals from water. Some studies reported the treatment of the pharmaceuticals by ozonation [19, 20], GAC adsorption [21, 22], and NF/RO membrane processes [23]. There are also studies that reported the treatment of PFASs by ozonation [24, 25], GAC adsorption [26], and NF/RO membrane processes [27, 28]. Furthermore, Appleman et al. and Flores et al. reported the treatment of PFASs in American and Spanish water treatment plants that adopted conventional and advanced water treatment processes, respectively [29, 30]. However, the pollutants have been detected even in drinking water treatment plants equipped with such methods. In addition, studies that comprehensively analyze their removal efficiency according to the operating conditions such as temperature and $\mathrm{pH}$ are still incomplete. Moreover, it has been rarely reported that studies compare the treatment efficiency of pollutants by a major advanced water treatment process targeting the same MPs in parallel in a single paper and examine the possibility of mutually complementing the removal efficiency according to the process. Herein, we examined the removal efficiencies of the four most frequently detected pharmaceuticals (carbamazepine, crotamiton, metformin, and sulfamethoxazole) and PFASs (PFHxA, PFHxS, PFOA, and PFOS) in parallel by lab-scale advanced water treatment processes-ozone oxidation, granular activated carbon (GAC) adsorption, and reverse osmosis (RO) membrane processes by varying two major operating conditions: $\mathrm{pH}$ and temperature. The physicochemical properties of the target chemicals, such as $\mathrm{pK}_{\mathrm{a}}, \log \mathrm{K}_{\mathrm{OW}}$, and molecular geometry were also correlated with the removal efficiencies. We believe that our findings can help to optimize the removal efficiency of MPs through an appropriate combination of advanced water treatment processes in the future.

\section{Materials and Methods}

\subsection{Chemical and Materials}

PFASs (PFHxA, PFHxS, PFOA, and PFOS) were purchased from Wellington Laboratories (Guelph, Canada). Four isotope-labeled compounds (sodiumperfluoro-1-hexane $\left[{ }^{18} \mathrm{O}_{2}\right]$ sulfonate, sodiumperfluoro$1-\left[1,2,3,4-{ }^{13} \mathrm{C}_{4}\right]$ octane sulfonate, perfluoro-n- $\left[1,2-{ }^{13} \mathrm{C}_{2}\right]$ hexanoic acid, perfluoro-n-[1,2,3,4- $\left.{ }^{13} \mathrm{C}_{4}\right]$ octanoic acid) were purchased from Wellington Laboratories and used as internal standards. Four pharmaceuticals (carbamazepine (CBZ), crotamiton (CTM), metformin
(MF), and sulfamethoxazole (SMZ)) were purchased from Sigma-Aldrich (St. Louis, MO, USA). Two isotope-labeled pharmaceuticals (metformin- $\mathrm{d}_{6}$, sulfamethoxazole- ${ }^{13} \mathrm{C}_{6}$ ) were purchased from Sigma-Aldrich and used as internal standards. Milli-Q water and organic solvents that were used for the analysis of the target compounds such as methyl alcohol, acetone, and acetonitrile were purchased from J.T. Baker's (Phillipsburg, NJ, USA); HPLC grade or higher reagents were used. Potassium indigo trisulfonate, phosphoric acid, and sodium thiosulfate $\left(\mathrm{Na}_{2} \mathrm{~S}_{2} \mathrm{O}_{3}\right)$ were obtained from Sigma-Aldrich. All chemicals were used as received.

\subsection{Ozonation Process}

An ozone generator (Triogen LAB2B, Scotland) was used to generate an ozone stock solution by passing pure oxygen gas (KOSEM, South Korea) and bubbling the ozone-containing gas into $1 \mathrm{~L}$ of deionized (DI) water chilled using an ice-bath. The absorbance of a mixture of indigo blue solution at $600 \mathrm{~nm}$ was measured to determine the concentration of ozone stock solution using a spectrophotometer (Optizen POP, South Korea); the concentration was calculated using the following equation [31]. The concentration of the ozone stock solution was determined by the Eq. (1) measuring the absorbance of the mixture of indigo blue solution at $600 \mathrm{~nm}$.

$$
m g O_{3} / L=\frac{\left(\mathrm{A}_{\mathrm{DIL}}-\mathrm{A}_{\mathrm{OZONE}}\right)}{f \times b} \times(\text { Dilution factor })
$$

$A_{D L}, A_{O Z O N E}, f$, and $b$ denote the absorbance of the diluted solution, absorbance of ozone stock added solution, indigo blue solution sensitivity coefficient (0.42), and path length of the cell $(\mathrm{cm})$, respectively. Indigo blue solution was prepared by mixing $1 \mathrm{~mL}$ of phosphoric acid, $770 \mathrm{mg}$ of potassium indigo trisulfonate, and filled up with DI water to $1 \mathrm{~L}$. The prepared indigo blue solution was stored in a dark and used within a month. The reaction was conducted in a $30 \mathrm{~mL}$ batch reactor under gentle stirring. The experiments were carried out with mixture of pharmaceuticals and mixture of PFASs, respectively. The concentrations of each target compound were $100 \mathrm{ng} / \mathrm{mL}$. The ozone stock solution was spiked into the ozonation reactor to a concentration of $1 \mathrm{mg} / \mathrm{L}$. The reaction lasted $3 \mathrm{~min}$, after which sodium thiosulfate and an ozone scavenger were added to the sample to remove the residual ozone. Then, the concentrations of the target material were analyzed by liquid chromatography-mass spectrometry (LC-MS). Additionally, $1 \mathrm{M} \mathrm{HCl}$ and 1 $\mathrm{M} \mathrm{NaOH}$ were used to adjust the $\mathrm{pH}$ to 3 and 11 .

\subsection{GAC Adsorption Process}

The GAC used during the experiments was Filtrasorb 400 (Calgon Carbon Corporation, Belgium), a highly active granular mesoporous carbon for removing dissolved organic compounds during the water treatment process. The GAC was sonicated until the black powder was removed. GAC adsorption experiments were carried out upflow in a $1 \mathrm{~cm}$-diameter double-walled glass column packed with a total bed volume of $0.36 \mathrm{~mL}$ of GAC (1.80 g in weight). The remaining space was filled with glass beads. The GAC system was operated at an empty bed contact time of $100 \mathrm{~s}$ and flow rate of $0.6 \mathrm{~mL}$ $\mathrm{min}^{-1}$. Samples were collected $90 \mathrm{~min}$ after the start of the experiments. The concentrations of target compounds were analyzed 


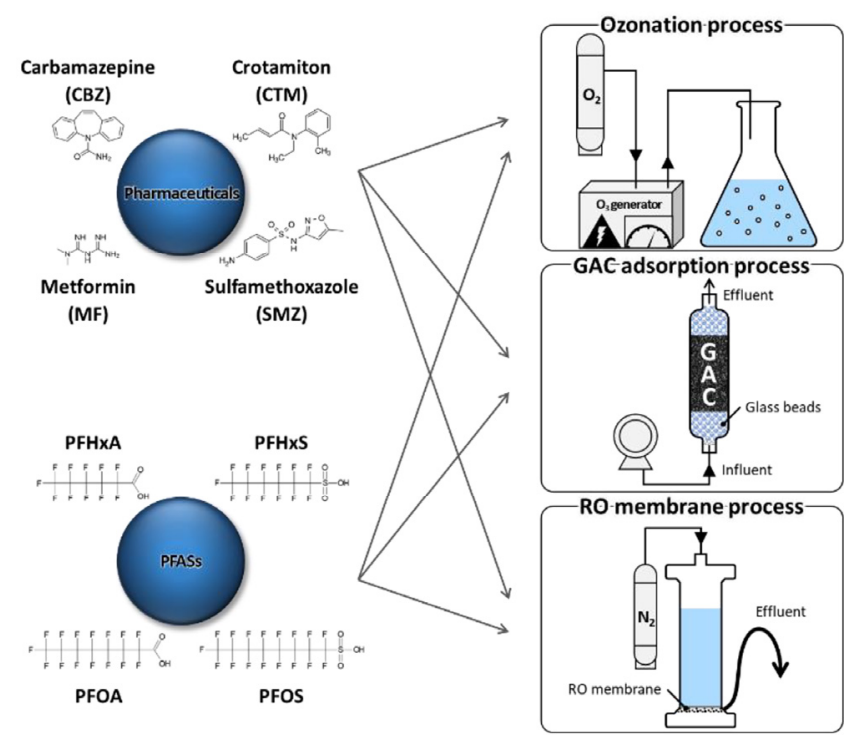

Fig. 1. Schematic of advanced water treatment processes and target micropollutants.

by LC-MS. Each target compound was spiked with DI water to a concentration of $100 \mathrm{ng} / \mathrm{mL} ; 1 \mathrm{M} \mathrm{HCl}$ and $1 \mathrm{M} \mathrm{NaOH}$ were used to adjust the $\mathrm{pH}$ to 3 and 11 .

\subsection{RO membrane Process}

The filtration experiments were carried out in a stainless-steel cell (HP4750, Sterlitech, USA) at a volume of $300 \mathrm{~mL}$. Two commercial RO membranes were used in the experiments: TW 80 for household water treatment and BW ES for brackish water treatment were supplied by LG water solutions (South Korea). Membrane coupons were cut with an effective membrane area of $19.6 \mathrm{~cm}^{2}$. Before being loaded into the filtration cell, the membranes were fully rinsed with DI water and then soaked in DI water for over $24 \mathrm{~h}$. The experiments were conducted with mixture of pharmaceuticals and mixture of PFASs, respectively; the feed concentrations of each target compound were $100 \mathrm{ng} / \mathrm{mL}$. The pressure was applied with pure nitrogen gas to $8 \mathrm{bar}$ at $25^{\circ} \mathrm{C}$. Samples were collected $10 \mathrm{~min}$ after the start of the experiments and analyzed by LC-MS.

\subsection{Analytical Methods}

The concentrations of PFASs and pharmaceuticals in the extracts were determined using an Agilent 1200 HPLC system coupled to an Agilent 6460 electrospray triple-quadrupole mass spectrometer (Agilent Technologies, Santa Clara, CA, USA) using a method described in previous publications [32]. A binary mobile phase gradient was used and the relevant compounds were identified and quantified using multiple reaction monitoring methods. The HPLC system was fitted with a guard column (4.6 mm id, $12.5 \mathrm{~mm}$ long, $5 \mu \mathrm{m}$ particle diameter; Agilent Technologies) and a ZORBAX XDB C18 HPLC column (4.6 mm id, $150 \mathrm{~mm}$ long, $3.5 \mu \mathrm{m}$ particle diameter; Agilent Technologies). Detailed analytical conditions and conditions used to quantify pharmaceuticals and PFASs are referred to in previous research [32].

\section{Results and Discussion}

\subsection{Treatment Efficiencies of Target Compounds by Ozonation}

The removal efficiencies of target compounds by the ozonation process are shown in Fig. 2. The experiments were carried out at two different temperatures $\left(5^{\circ} \mathrm{C}\right.$ and $\left.25^{\circ} \mathrm{C}\right)$ to simulate water treatment plants in winter and summer. In addition, the effect of hydrogen peroxide $\left(\mathrm{H}_{2} \mathrm{O}_{2}\right)$, which is known to enhance ozonation efficiency, was evaluated. It was found that CBZ, CTM, and SMZ were fully eliminated (>99\%) within $10 \mathrm{~s}$ of reaction, whereas MF and all PFASs showed significantly lower removal efficiencies. This is thought to be due to the chemically stable structure of MF and PFASs. In particular, PFASs have extremely high thermal and chemical stabilities induced by strong carbon-fluorine bonds. Fluorine is the most powerful electron-withdrawing element; as such, electron-withdrawing groups (e.g. fluorine, $-\mathrm{COOH}$, and $\left.-\mathrm{SO}_{3} \mathrm{H}\right)$ depress the reactivity of ozone [33]. Thus, PFASs, which have strong carbon-fluorine bonds together with electron-withdrawing functional groups, are resistant to strong oxidants such as ozone [1]. On the other hand, the change in removal efficiencies according to the reaction temperature or the addition of $\mathrm{H}_{2} \mathrm{O}_{2}$ was found to be insignificant. This indicates that the treatment efficiencies of target compounds by oxidation are mainly affected by the chemical stability of the target materials rather than the change in reaction conditions.

Removal efficiencies of target compounds in the ozonation process according to acidic ( $\mathrm{pH}$ 3), neutral ( $\mathrm{pH}$ 7), and basic ( $\mathrm{pH} 11)$ conditions are shown in Fig. 3. Pharmaceuticals such as CBZ, CTM, and SMZ were effectively removed both under acidic and neutral conditions, whereas removal efficiencies at basic conditions significantly decreased to less than $20 \%$. This is because hydroxyl ions $\left(\mathrm{OH}^{-}\right)$under basic conditions radically reacted with the ozone by a series of chain reactions, thus, ozone was rapidly consumed at the initial stage (Eq. (2)). Although hydroxyl radicals $(\mathrm{OH} \cdot)$ - more powerful oxidants than ozone-were generated during this step (Eq. (3)), they are extremely unstable and short-lived in water. Therefore, the generated hydroxyl radicals only reacted with the neighboring elements and immediately disappear [34]. Furthermore, the initial concentration of the target compounds was very low (100 ng/mL) and the generated hydroxyl radicals could not react sufficiently with the pollutants. In other words, even though hydroxyl radials are more oxidative than ozone, they are unsuitable for the ozonation process due to their extremely short lifetime.

$$
\begin{gathered}
\mathrm{O}_{3}+\mathrm{OH}^{-} \rightarrow \mathrm{HO}_{2}^{-}+\mathrm{O}_{2} \\
\mathrm{O}_{3}+\mathrm{HO}_{2}^{-} \rightarrow \mathrm{OH} \cdot+\mathrm{O}_{2} \cdot{ }^{-}+\mathrm{O}_{2}
\end{gathered}
$$

Meanwhile, MF and PFASs were hardly removed during the ozonation processes regardless of $\mathrm{pH}$. As PFASs have antioxidant properties for various processes such as oxidation, ozone $/ \mathrm{H}_{2} \mathrm{O}_{2}$, ozone/UV, and ozone/TiO ${ }_{2}$ processes, it is unsuitable to treat PFASs through chemical oxidation processes [24, 25]. 


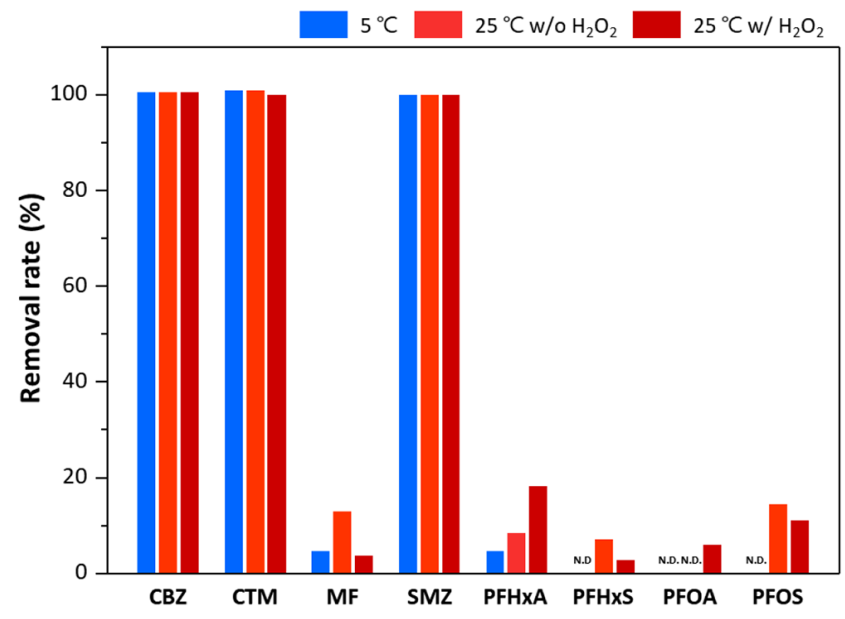

Fig. 2. Effects of temperature and $\mathrm{H}_{2} \mathrm{O}_{2}$ on the removal rates of pharmaceuticals and PFASs from ozonation process.

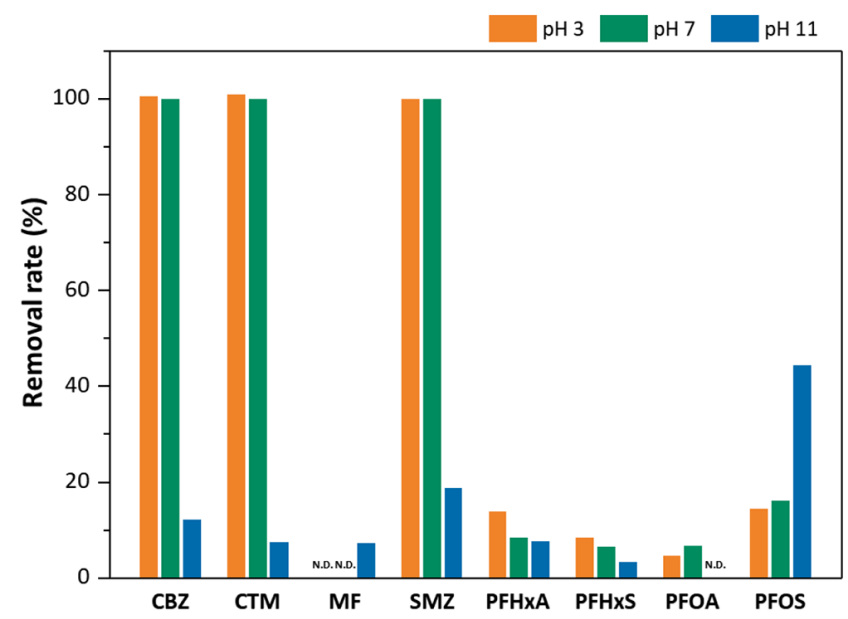

Fig. 3. Effects of $\mathrm{pH}$ on the removal rates of pharmaceuticals and PFASs from ozonation process.

\subsection{Treatment Efficiencies of Target Compounds by the GAC Adsorption Process}

Removal efficiencies of target compounds in the GAC adsorption processes at temperatures of $5^{\circ} \mathrm{C}$ and $25^{\circ} \mathrm{C}$ are shown in Fig. 4, assuming that these are the winter and summer temperatures of water treatment plants, respectively. Not only pharmaceuticals including CBZ, CTM, and SMZ, but also PFASs, which were rarely removed in the ozonation process, were effectively treated (> 99.5\%) by the GAC adsorption processes. The effect of temperature was insignificant. MF remained unaffected after the GAC process, which is thought to be due to the extremely high hydrophilicity of MF $\left(\log \mathrm{K}_{\mathrm{OW}}=-2.64\right.$ at $\mathrm{pH} 7$ ), as shown in Table 1. GAC is a porous material with a hydrophobic surface and high adsorption rate for hydrophobic materials, whereas selective physical adsorption occurs for a hydrophilic material, thereby decreasing the adsorption rate [35]. The low removal efficiency of MF by the GAC process improved to $30 \%$ at $25^{\circ} \mathrm{C}$. In general, the adsorption reaction is considered to be an exothermic reaction and the adsorption capacity decreases with an increase in temperature [36, 37]. In this study, however, the removal efficiency of $\mathrm{MF}$ was improved with an increase in temperature. This phenomenon is thought to be due to the activation of new adsorption sites and accelerated movement of MF molecules at high temperatures [38].

The removal efficiencies of target compounds in the GAC adsorption process according to acidic $(\mathrm{pH} 3)$, neutral ( $\mathrm{pH} 7$ ), and basic (pH 11) conditions were also assessed (Fig. 5). The treatment efficiencies of all the tested PFASs and pharmaceuticals except MF remained largely unaffected by variations in $\mathrm{pH}$. Interestingly, the low treatment efficiency of $\mathrm{MF}$ at lower $\mathrm{pH}(<10 \%$ at $\mathrm{pH} 3)$ increased as the $\mathrm{pH}$ increased and reached more than $95 \%$ at $\mathrm{pH} 11$. This may be because the hydrophobicity of MF dramatically changed along with $\mathrm{pH}$. As depicted in Fig. 6, MF has two $\mathrm{pK}_{\mathrm{a}}$ values (2.8 and 11.5). Between acidic and neutral $\mathrm{pH}, \mathrm{MF}$ molecules have a positive charge due to the protonated form of amine groups, making them hydrophilic under these conditions. As $\mathrm{pH}$ approaches $\mathrm{pK}_{\mathrm{a} 2}$ (11.5), the deprotonated form of MF becomes dominant, thus, the molecule develops hydrophobic characteristics. Considering

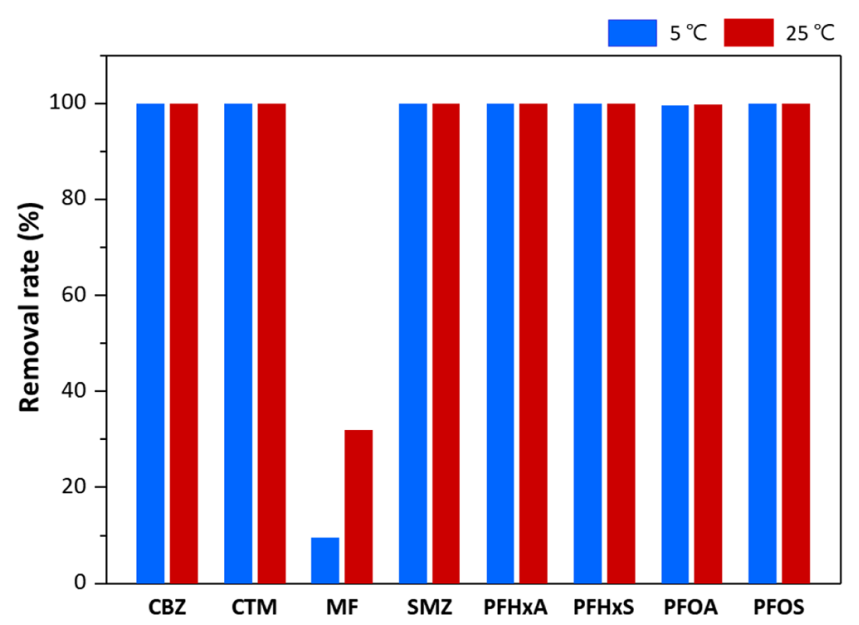

Fig. 4. Effects of temperature on the removal rates of pharmaceuticals and PFASs from GAC adsorption process.

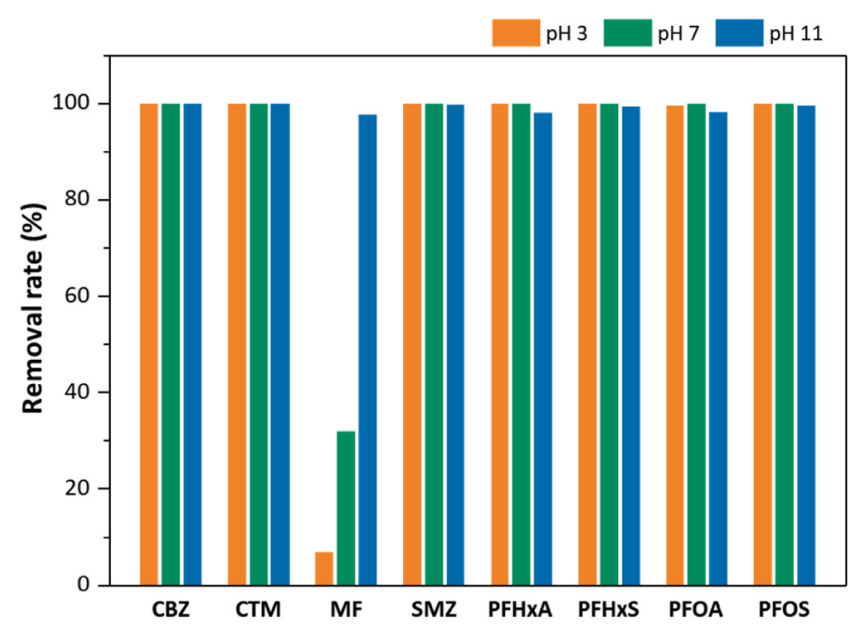

Fig. 5. Effects of $\mathrm{pH}$ on the removal rates of pharmaceuticals and PFASs from GAC adsorption process. 
Table 1. Physicochemical Properties of The Pharmaceuticals and PFASs

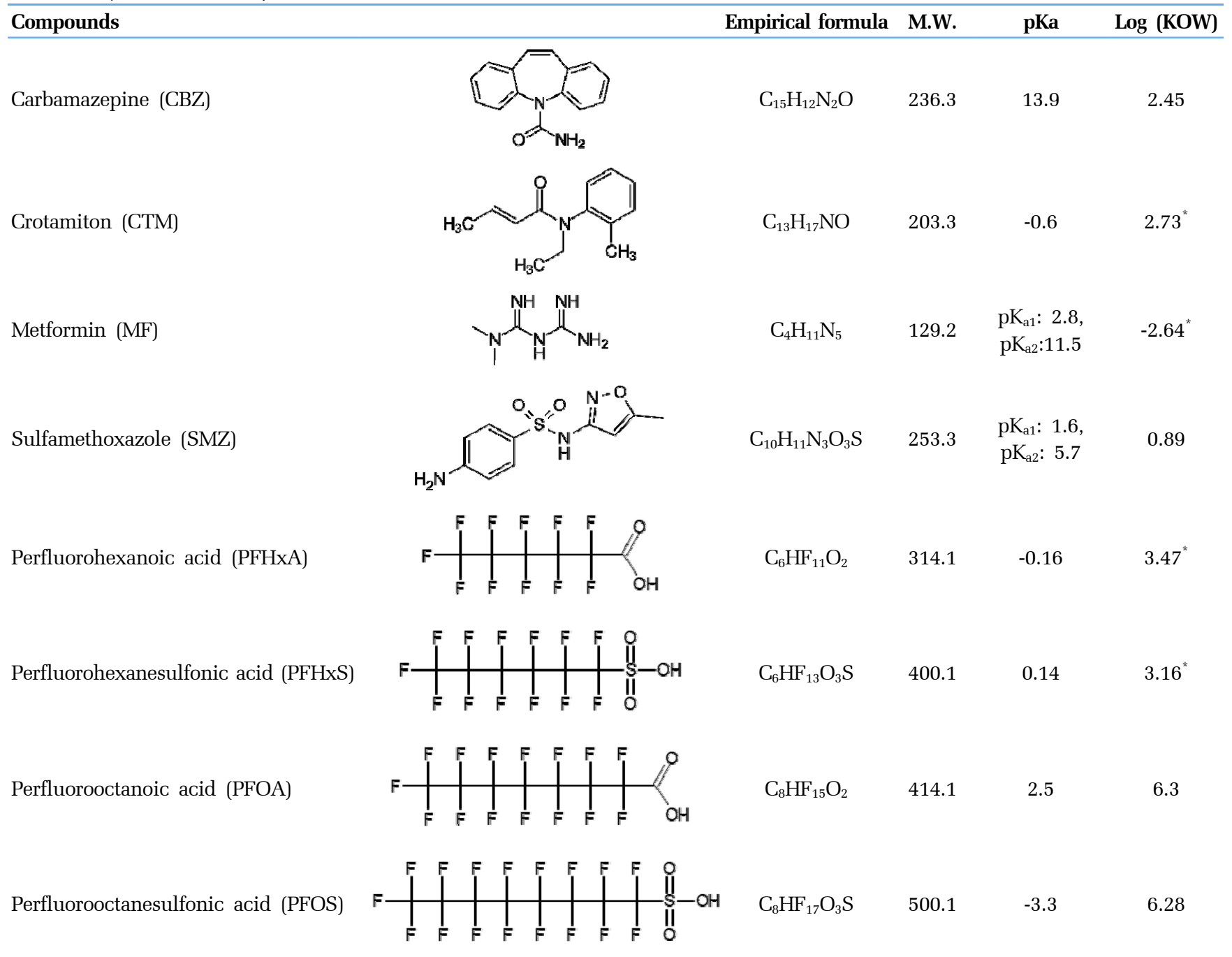

${ }^{*}$ : estimated value

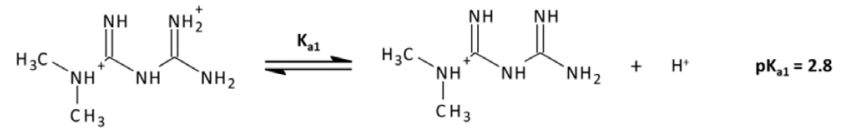

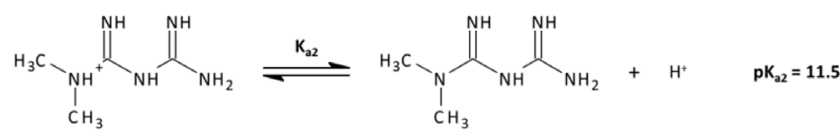

Fig. 6. Protonation of metformin molecule according to $\mathrm{pH}$ change.

that ozonation failed to remove MF, $\mathrm{pH}$ control could be a strategy to improve the removal rate of MF by a simple method in the GAC process.

\subsection{Treatment Efficiencies of Target Compounds by RO Membrane Process}

Rejection of pharmaceuticals and PFASs in the RO membrane proc- ess is shown in Fig. 7(a). Two RO membranes (TW 80 and BW ES) were used in this study: TW 80 membrane was designed to treat tap water which contains a low concentration of impurities, whereas BW ES membrane was designed to treat brackish water which contains much higher concentration of salts and pollutants. The experimenting pressure of 8 bars followed the average operating pressure of the household RO membrane. BW ES showed higher rejection rates compared to TW 80 under the same operating pressure for all target compounds, indicating that BW ES has a denser skin layer than TW 80. It is also consistent with the fact that BW ES provides higher $\mathrm{NaCl}$ rejection (99.6\%) than TW 80 (98\%), as provided by the manufacturer.

The rejection rates of MF with the small molecular weight ( $\sim 130$ Da) and highly hydrophilic properties $\left(\log \mathrm{K}_{\mathrm{OW}}=-2.64\right.$ at $\left.\mathrm{pH} 7\right)$ were found to be remarkably low ( 10\%). However, other pharmaceuticals (CBZ, CTM, SMZ) showed a far higher rejection (>99\%) compared to PFASs (60 90\%). This is an interesting result 

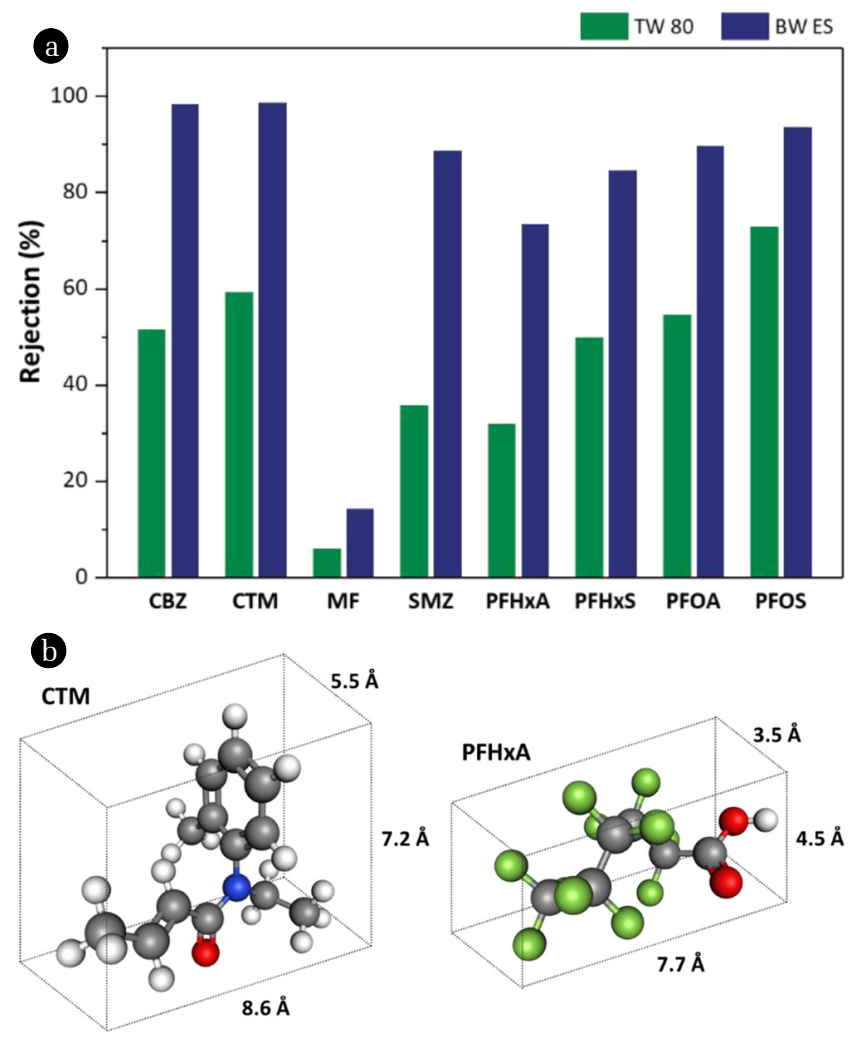

Fig. 7. (a) Rejection of pharmaceuticals and PFASs by varying RO membranes. (b) 3-dimensional molecular size of CTM and PFHxA.

considering the fact that the molecular weight of pharmaceuticals $(200 \sim 250 \mathrm{Da})$ is smaller than that of PFASs (300 500 Da). We speculate that this is due to the difference in the steric partitioning factors such as molecular length, width, and shape. As shown in Fig. 7(b), CBZ, CTM, and SMZ have two- or three-dimensional bulky molecular structures, whereas PFASs have a one-dimensional linear structure. Thereby, PFASs could pass through the RO membrane in the longitudinal direction, while the pharmaceuticals were blocked due to their bulky structure. Similar results have been also reported in other studies [39-41]. On the other hand, in comparison to the PFASs, it was clearly observed that the rejection was increased as the molecular weight was increased, implying that an overall molecular size is still important in the PFASs rejection. The above results show that there is a limit to predict the rejection of pollutants by the RO membrane simply considering molecular weight, thereby molecular geometry must be considered.

\section{Conclusions}

In this study, the treatment efficiencies of four pharmaceuticals and four PFASs that are frequently detected in the water systems were assessed by three advanced water treatment processes: ozonation, GAC adsorption, and RO membrane processes. CBZ, CTM, and SMZ were effectively treated by the ozonation process, whereas
MF and PFASs were unaffected, regardless of the temperature. Under basic conditions, the lifetime of ozone and treatment efficiency decreased. MF and PFASs were evaluated to have low treatment efficiency by ozone oxidation, regardless of temperature and $\mathrm{pH}$ owing to their chemically stable structures. Not only pharmaceuticals such as CBZ, CTM, and SMZ but also PFASs were effectively removed by the GAC adsorption process regardless of temperature and $\mathrm{pH}$. MF was hardly removed under acidic and neutral conditions. As the $\mathrm{pH}$ increased, however, MF lost electric charge and developed increased hydrophobicity; thus, treatment efficiency was improved via GAC. In the RO membrane process, at the same pressure, the RO membrane for brackish water showed higher removal efficiencies for all target substances than for the residential water. Even in the RO process, MF showed a significant low rejection $(<10 \%)$ due to its small molecular weight and a high hydrophilicity, while other pharmaceuticals had high rejection rates of $98 \%$ or more. PFASs having a one-dimensional molecular structure exhibited a lower level of rejection than pharmaceuticals despite of having a higher molecular weight.

This study proved that the GAC adsorption process is very effective for treating extremely persistent PFASs regardless of temperature and $\mathrm{pH}$ conditions. Furthermore, it was confirmed that substances such as MF that are not generally treated by ozonation and GAC processes can be treated by adjusting the $\mathrm{pH}$.

\section{Acknowledgement}

This work was supported by a 2-year research grant from Pusan National University.

\section{Author Contributions}

H.S.C. (Ph. D. student) conducted the target compounds removal experiments and wrote the manuscript. K.Y.K. (Researcher) conducted the analytical experiments and wrote the manuscript. J.E.O. (Professor) revised the manuscript. J.H.K. (Associate Professor) wrote and revised the manuscript.

\section{References}

1. Kümmerer K. The presence of pharmaceuticals in the environment due to human use-present knowledge and future challenges. J. Environ. Manage. 2009;90:2354-2366.

2. Neale PA, Ait-Aissa S, Brack W, et al. Linking in vitro effects and detected organic micropollutants in surface water using mixture-toxicity modeling. Environ. Sci. Technol. 2015;49: 14614-14624.

3. Shao Y, Chen Z, Hollert H, Zhou S, Deutschmann B, Seiler TB. Toxicity of 10 organic micropollutants and their mixture: Implications for aquatic risk assessment. Sci. Total Environ. 2019;666:1273-1282.

4. Rahman MF, Peldszus S, Anderson WB. Behaviour and fate of perfluoroalkyl and polyfluoroalkyl substances (PFASs) in drinking water treatment: A review. Water Res. 2014;50:318-340. 
5. Park H, Choo G, Kim H, Oh JE. Evaluation of the current contamination status of PFASs and OPFRs in South Korean tap water associated with its origin. Sci. Total Environ. 2018;634: 1505-1512

6. Tröger R, Klöckner P, Ahrens L, Wiberg K. Micropollutants in drinking water from source to tap - Method development and application of a multiresidue screening method. Sci. Total Environ. 2018;627:1404-1432.

7. Kim H, Ekpe OD, Lee JH, Kim DH, Oh JE. Field-scale evaluation of the uptake of Perfluoroalkyl substances from soil by rice in paddy fields in South Korea. Sci. Total Environ. 2019;671:714-721.

8. Luo Y, Guo W, Ngo HH, et al. A review on the occurrence of micropollutants in the aquatic environment and their fate and removal during wastewater treatment. Sci. Total Environ. 2014;473-474:619-641.

9. Dietrich S, Ploessl F, Bracher F, Laforsch C. Single and combined toxicity of pharmaceuticals at environmentally relevant concentrations in Daphnia magna - A multigenerational study. Chemosphere 2010;79:60-66.

10. West PR, Weir AM, Smith AM, Donley ELR, Cezar GG. Predicting human developmental toxicity of pharmaceuticals using human embryonic stem cells and metabolomics. Toxicol. Appl. Pharmacol. 2010;247:18-27.

11. Borg D, Lund BO, Lindquist NG, Håkansson H. Cumulative health risk assessment of 17 perfluoroalkylated and polyfluoroalkylated substances (PFASs) in the Swedish population. Environ. Int. 2013;59:112-123.

12. Neale PA, Leusch FDL, Escher BI. Applying mixture toxicity modelling to predict bacterial bioluminescence inhibition by non-specifically acting pharmaceuticals and specifically acting antibiotics. Chemosphere 2017;173:387-394.

13. Menger F, Pohl J, Ahrens L, Carlsson G, Örn S. Behavioural effects and bioconcentration of per- and polyfluoroalkyl substances (PFASs) in zebrafish (Danio rerio) embryos. Chemosphere 2020;245:125573.

14. Cizmas L, Sharma VK, Gray CM, McDonald TJ. Pharmaceuticals and personal care products in waters: occurrence, toxicity, and risk. Environ. Chem. Lett. 2015;13:381-394.

15. de Jesus Gaffney V, Almeida CMM, Rodrigues A, Ferreira E, Benoliel MJ, Cardoso VV. Occurrence of pharmaceuticals in a water supply system and related human health risk assessment. Water Res. 2015;72:199-208.

16. Scher DP, Kelly JE, Huset CA, et al. Occurrence of perfluoroalkyl substances (PFAS) in garden produce at homes with a history of PFAS-contaminated drinking water. Chemosphere 2018;196: 548-555.

17. Marchetti N, Guzzinati R, Catani M, Massi A, Pasti L, Cavazzini A. New insights into perfluorinated adsorbents for analytical and bioanalytical applications. Anal. Bioanal. Chem. 2015;407: 17-21.

18. Liu S, Yang R, Yin N, Faiola F. The short-chain perfluorinated compounds PFBS, PFHxS, PFBA and PFHxA, disrupt human mesenchymal stem cell self-renewal and adipogenic differentiation. Int. J. Environ. Sci. 2020;88:187-199.

19. Nakada N, Shinohara H, Murata A, et al. Removal of selected pharmaceuticals and personal care products (PPCPs) and endocrine-disrupting chemicals (EDCs) during sand filtration and ozonation at a municipal sewage treatment plant. Water Res. 2007;41:4373-4382.

20. Alharbi SK, Price WE, Kang J, Fujioka T, Nghiem LD. Ozonation of carbamazepine, diclofenac, sulfamethoxazole and trimethoprim and formation of major oxidation products. Desalin. Water Treat. 2016;57:1-12.

21. Yu Z, Peldszus S, Huck PM. Adsorption characteristics of selected pharmaceuticals and an endocrine disrupting compound -Naproxen, carbamazepine and nonylphenol—on activated carbon. Water Res. 2008;42:2873-2882.

22. Qiao T, Yu Z, Zhang X, Au DWT. Occurrence and fate of pharmaceuticals and personal care products in drinking water in southern China. J. Environ. Monit. 2011;13:3097-3103.

23. Phadunghus K, Wongrueng A, Rakruam P, Wattanachira S, Punyapalakul P. Efficiencies of $\mathrm{NF}$ and $\mathrm{RO}$ membranes on pharmaceutical removal and membrane fouling effects. Eng. J. 2017;21:101-112.

24. Schröder HF, Meesters RJW. Stability of fluorinated surfactants in advanced oxidation processes - A follow up of degradation products using flow injection-mass spectrometry, liquid chromatography-mass spectrometry and liquid chromatography-multiple stage mass spectrometry. J. Chromatogr. A 2005;1082:110-119.

25. Huang J, Wang X, Pan Z, Li X, Ling Y, Li L. Efficient degradation of perfluorooctanoic acid (PFOA) by photocatalytic ozonation. Chem. Eng. J. 2016;296:329-334.

26. Hansen MC, Børresen MH, Schlabach M, Cornelissen G. Sorption of perfluorinated compounds from contaminated water to activated carbon. J. Soils Sediments. 2010;10:179-185.

27. Tang CY, Fu QS, Robertson AP, Criddle CS, Leckie JO. Use of reverse osmosis membranes to remove perfluorooctane sulfonate (pfos) from semiconductor wastewater. Environ. Sci. Technol. 2006;40:7343-7349.

28. Tang CY, Fu QS, Criddle CS, Leckie JO. Effect of flux (transmembrane pressure) and membrane properties on fouling and rejection of reverse osmosis and nanofiltration membranes treating perfluorooctane sulfonate containing wastewater. Environ. Sci. Technol. 2007;41:2008-2014.

29. Flores C, Ventura F, Martin-Alonso J, Caixach J. Occurrence of perfluorooctane sulfonate (PFOS) and perfluorooctanoate (PFOA) in N.E. Spanish surface waters and their removal in a drinking water treatment plant that combines conventional and advanced treatments in parallel lines. Sci. Total Environ. 2013;461-462:618-626.

30. Appleman T D, Higgins C P, Quiñones O, Vanderford B J, Kolstad C, Zeigler-Holady J C, Dickenson E R V. Treatment of polyand perfluoroalkyl substances in U.S. full-scale water treatment systems. Water Res. 2014;51:246-255.

31. Gordon G, Bubnis B. Residual Ozone Measurement: Indigo Sensitivity Coefficient Adjustment. Ozone: Sci. Eng. 2002;24:17-28.

32. Kim KY, Ekpe OD, Lee HJ, Oh JE. Perfluoroalkyl substances and pharmaceuticals removal in full-scale drinking water treatment plants. J. Hazard. Mater. 2020;400:123235.

33. von Gunten U. Ozonation of drinking water: Part I. Oxidation kinetics and product formation. Water Res. 2003;37:1443-1467.

34. Gardoni D, Vailati A, Canziani R. Decay of Ozone in Water: A Review. Ozone: Sci. Eng. 2012;34:233-242. 
35. Khan M H, Ha DH, Jung J. Optimizing the industrial wastewater pretreatment by activated carbon and coagulation: Effects of hydrophobicity/hydrophilicity and molecular weights of dissolved organics. J. Environ. Sci. Health, Part A: Toxic/Hazard. Subst. Environ. Eng. 2013;48:534-542.

36. Zhou X, Yi H, Tang X, Deng H, Liu H. Thermodynamics for the adsorption of $\mathrm{SO}_{2}, \mathrm{NO}$ and $\mathrm{CO}_{2}$ from flue gas on activated carbon fiber. Chem. Eng. J. 2012;200-202:399-404.

37. Zhang J. Phenol Removal from Water with Potassium Permanganate Modified Granular Activated Carbon. J. Environ. Prot. 2013;04:411-417.

38. Sudhakar P, Mall ID, Srivastava VC. Adsorptive removal of bisphenol-A by rice husk ash and granular activated carbon-A comparative study. Desalin. Water Treat. 2016;57:12375-12384

39. Kiso Y, Kitao T, Jinno K, Miyagi M. The effects of molecular width on permeation of organic solute through cellulose acetate reverse osmosis membranes. J. Membr. Sci. 1992;74:95-103.

40. Agenson $\mathrm{KO}$, Oh J, Urase T. Retention of a wide variety of organic pollutants by different nanofiltration/reverse osmosis membranes: controlling parameters of process. J. Membr. Sci. 2003;225:91-103.

41. Kiso Y, Muroshige K, Oguchi T, et al. Effect of molecular shape on rejection of uncharged organic compounds by nanofiltration membranes and on calculated pore radii. J. Membr. Sci. 2010;358:101-113. 